\title{
Measurement of Mechanical Strain using Chromatic Monitoring of Photoelasticity
}

\author{
Garza C. ${ }^{1, a}$, Deakin A.G. ${ }^{2, b}$, Jones G. R. ${ }^{2, c}$, Spencer J. W. ${ }^{2, d}$ \\ and Hon K.K.B. ${ }^{1, e}$ \\ ${ }^{1}$ Department of Engineering, The University of Liverpool, Brownlow Hill, Liverpool, L69 3GH, UK \\ ${ }^{2}$ CIMS, Centre for Intelligent Monitoring Systems Research Group, Department of Electrical \\ Engineering and Electronics, Brownlow Hill, Liverpool, L69 3GJ, UK \\ a claudia.garza@liverpool.ac.uk, ${ }^{\mathrm{b}}$ anthonyd@liverpool.ac.uk, ${ }^{\mathrm{c}}$ grjones@liverpool.ac.uk, \\ djoe@liverpool.ac.uk, ${ }^{e}$ honkk@liverpool.ac.uk
}

Keywords: Chromatic monitoring, photo-elasticity, Strain Measurement

\begin{abstract}
The present contribution describes a chromatic processing approach for quantifying the two dimensional, polychromatic interference patterns produced by a strained photo-elastic element and recorded with a CCD camera. The outputs from the three R, G, B channels of the camera covering a selected area of the interference pattern are processed to yield three chromatic parameters which are $\mathrm{H}$ (dominant signal wavelength), L (nominal signal strength), S (effective wavelength spread of signal). It is shown that the value of each of the three parameters varies with strain in a quasi cyclical manner, all being out of phase with each other. Consequently the strain measurement range and sensitivity can both be optimized by the use of the appropriate chromatic parameter within different strain ranges.
\end{abstract}

Introduction. There are several experimental methods for measuring strain such as foil strain gauges [1] split Hopkinson pressure bar (SHPB) or Kolsky bar technique [2], velocity interferometers [3] etc. There are also several optical methods such as the caustics method [4] Moiré techniques (interferometry or photography), speckle interferometry, photo-elasticity [5], etc. The latter may be deployed in one of several forms, e.g. Tardy method, use of compensators, digital photoelasticity [6] or chromatic evaluation of optical fibre based photo-elastic elements [7]. More recently Madhu and Ramesh [8] have considered colour adaptation in three fringe photoelasticity. The present paper is concerned with a further evaluation of the chromatic addressing [9] of photoelastic elements subjected to mechanical strain. A detailed investigation is made of the strain levels over which each of three parameters $(\mathrm{H}, \mathrm{L}, \mathrm{S})$ produced by chromatic processing [9] can be used to advantage for optimizing the measurement range and the sensitivity. For this purpose use has been made of two dimensional, polychromatic, photo-elastic interference patterns recorded with a CCD camera.

Measurement System. The experimental system used for the present tests was based upon monitoring the mechanical strain in a cantilever beam when loaded to various extents (Fig. 1). A polychromatic light source (Tungsten halogen lamp) was used to illuminate a $3 \mathrm{~mm}$ thick photoelastic element (PS-1A [10]) bonded to the strained beam with PC-1 Vishay Epoxy [10] resin adhesive. Images of the polychromatic fringes produced by the strained photo-elastic element were obtained using a 7 MegaPixels CCD camera in conjunction with an optical polarizing filter. The outputs from the CCD camera were transferred to a personal computer for chromatic processing. Simultaneous to capturing the images of the photo-elastic fringes, the corresponding strain values to which the beam was exposed were recorded with a strain gauge bonded with M-BOND 200 Vishay Adhesive [10] to the beam which was situated under the beam and below the photo-elastic sensing element (Fig. 1). 




Figure 1.- Measurement System and Cantilever Beam

Chromatic Processing. A polychromatic signal produced by a photo-elastic element (e.g. Fig (2) interference signal) may be captured with a CCD camera via a matrix of three pixel detectors $(R, G$, B) at each spatial location and which have overlapping wavelength responses (Fig. 2)[9]. The outputs from the detectors $(\mathrm{r}, \mathrm{g}, \mathrm{b})$ can be transformed into three signal defining, chromatic parameters which are the dominating wavelength $(\mathrm{H})$, nominal signal strength $(\mathrm{L})$ and effective wavelength spread of the signal (1-S) [9]. Values of each of these chromatic parameters (H, L, S) may then be calibrated against strain values measured simultaneously with a strain gauge (Fig. 1). The algorithms used for the $\mathrm{r}, \mathrm{g}, \mathrm{b}$ transformation to $\mathrm{H}, \mathrm{L}, \mathrm{S}$ are given in Appendix I.

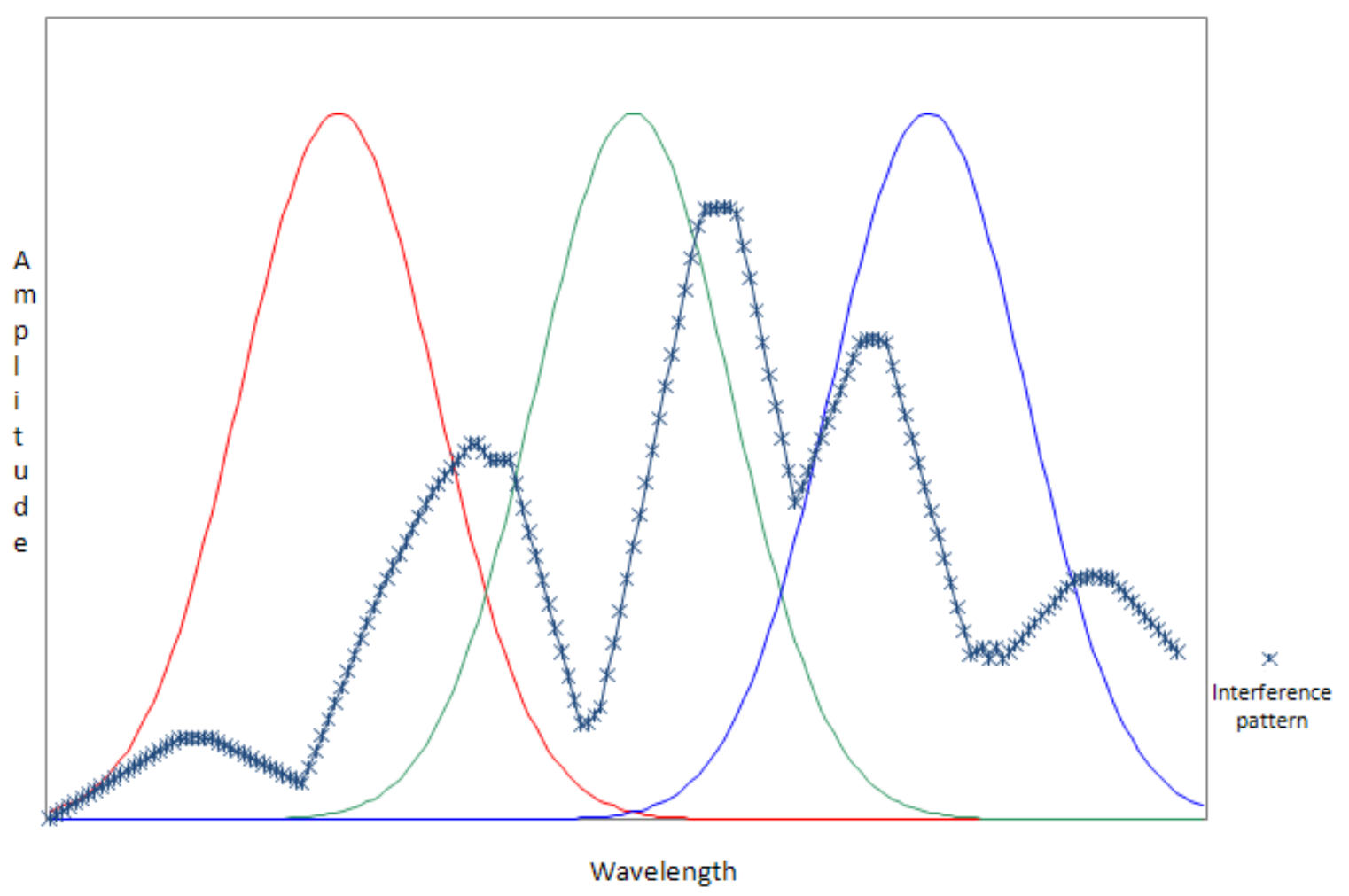

Figure 2.- Responses of 3 Non-Orthogonal Filters (R, G, B) as a function of wavelength superimposed upon a polychromatic signal $(\mathrm{X})$ 
Experimental procedure and calibration. The experimental procedure involved loading the cantilever to various extents and capturing the corresponding images of the polychromatic fringes produced by the strained photo-elastic element. The output values $\mathrm{r}, \mathrm{g}, \mathrm{b}$ obtained from each image pixel area were transferred to the computer and transformed into corresponding values of the chromatic parameters H, L, S for each pixel area using the algorithms given in Appendix I. Values of $\mathrm{H}, \mathrm{L}, \mathrm{S}$ were space averaged across a chosen sector of the image (314 pixel extent $\left[4 \mathrm{~mm}^{2}\right]$ over $20 * 20 \mathrm{~mm}$ actual area), using specially developed software (Droptri. [11]). This sector was located directly above the foil strain gauge which was situated under the beam and below the photo-elastic sensing element (Fig. 1). Examples of $\mathrm{H}, \mathrm{L}$ and $\mathrm{S}$ values determined in the above manner during a single test used for calibration are shown for different strain levels applied to the beam on Fig. 3 (a), (b), (c) respectively. These results exhibit quasi cyclic features with the maximum value plateau progressively shifted to higher strain values in order $\mathrm{H}, \mathrm{L}, \mathrm{S}$. In particular:

- $\mathrm{H}$ is insensitive to strain in the range $0-4.5 \mathrm{E}-05$ micro-strain but thereafter reduces monotonically $\left(20^{\circ}<\mathrm{H}<60^{\circ}\right)$ as the strain increases further.

- $\mathrm{L}$ is insensitive to strain in the range $5 \mathrm{E}-05-1.0 \mathrm{E}-04$ micro-strain but corresponds to two micro-strain values outside this range $(0.2<\mathrm{L}<0.8)$

- $\mathrm{S}$ is insensitive to strain in the range of $0-7.5 \mathrm{E}-05$ micro-strain but thereafter increases monotonically $(0.2<\mathrm{S}<0.42)$ as the strain increases further.

By using a look up table for the H, L, S parameters and strain measured with the strain gauge, strain calibrated values $\mathrm{Hm}, \mathrm{Lm}, \mathrm{Sm}$ for each of the three chromatic parameters can be obtained as indicated on the Flow Diagram of Fig. 4. The regions of high sensitivity for each parameter indicated on Fig. 3 can then be selected to provide a preferred strain value. As a result, the measurement range and sensitivity can both be optimized.



Figure 3.- Variation of Chromatic Parameters H, L, S with Measured Mechanical Strain (a) H: Strain (b) L: Strain (c) S: Strain 


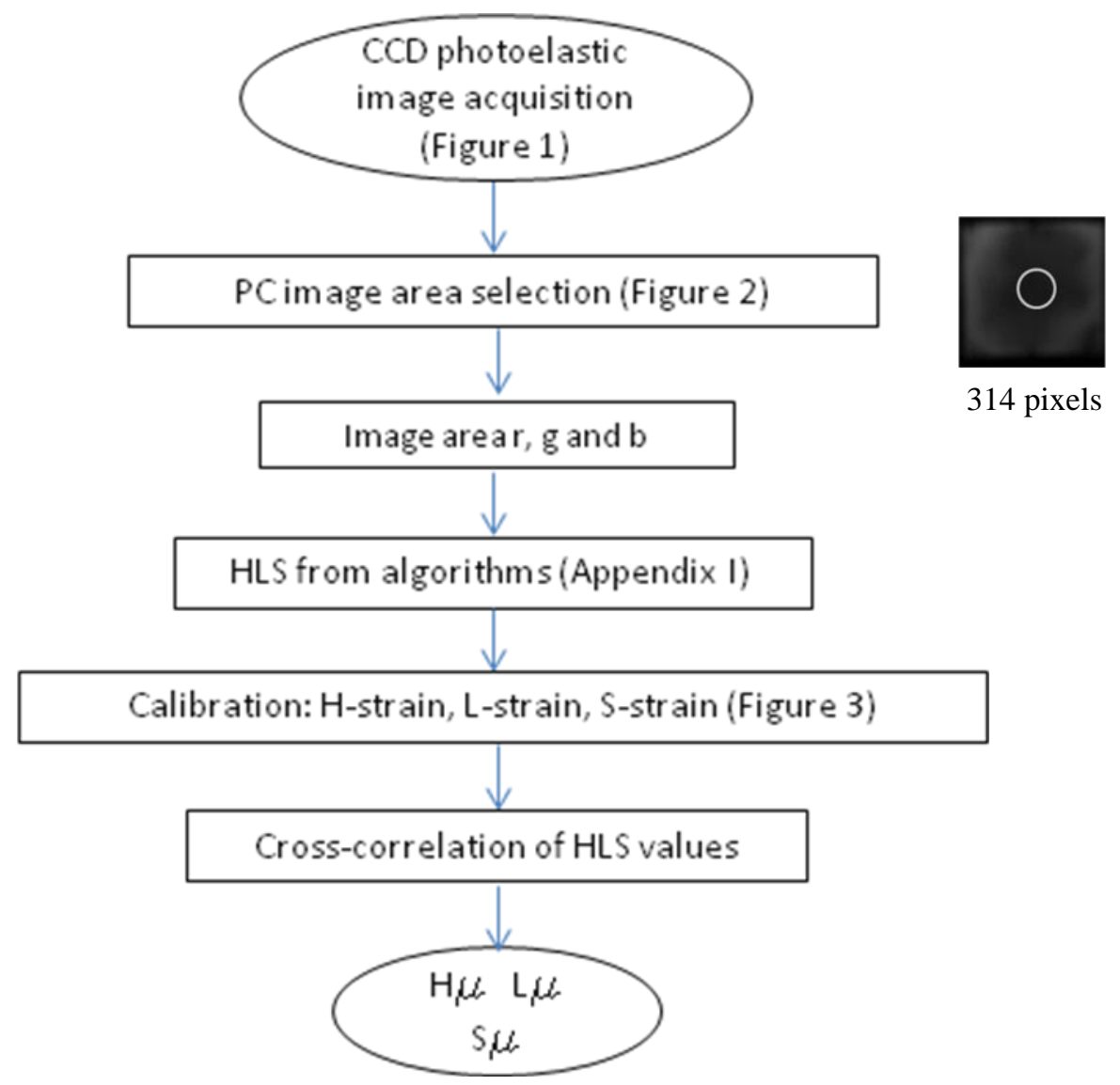

Figure 4.- Data Processing Flow Diagram for Obtaining Micro-strain from an Image of the Photo-elastic Interference Pattern

Experimental Results. Three additional tests were performed on the cantilever beam loaded to different extents. Values of H, L, S were determined from the resulting camera records. These were then converted into strain values ( $\mathrm{Hm}, \mathrm{Lm}, \mathrm{Sm}$ ) using the results from the calibration test (Fig. 3). The calculated strain values $(\mathrm{Hm}, \mathrm{Lm}, \mathrm{Sm})$ for a variety of imposed strains from the three different tests are shown (vertical axis) as a function of the imposed strain measured with the strain gauge (horizontal axis) on Fig. 5. Chromatic parameters results from each of the three tests are represented by different symbols as defined in the figure caption.

The results show an overall linear correlation between the chromatically derived (Hm, Lm, Sm) and the imposed strain values with a coefficient of determination $\mathrm{R}^{2}=0.955$ [12]. The scatter for each chromatic parameter is more pronounced in the strain range within which it exhibits low resolution (Fig. 3 (a), (b), (c)). i.e. at lower strain level for $\mathrm{Hm}(<4.5 \mathrm{E}-05)$ and $\mathrm{Sm}(<7.5 \mathrm{E}-05)$, but within the mid strain range for Lm (6 E-05 - 1.1 E-04) which shows highest scatter. Overall, the best behaved of the three chromatic parameters appears to be $\mathrm{Hm}$ with a coefficient of determination $\mathrm{R}^{2}=0.988$ compared with $\mathrm{R}^{2}=0.974,0.906$ for $\mathrm{Lm}$ and $\mathrm{Sm}$ respectively. Thus, a preferred option would be to rely upon the use of $\mathrm{Lm}$ at low strain values, $\mathrm{Hm}$ for the mid range and a combination of $\mathrm{Hm}, \mathrm{Lm}, \mathrm{Sm}$ for the high range (Fig. 5). 


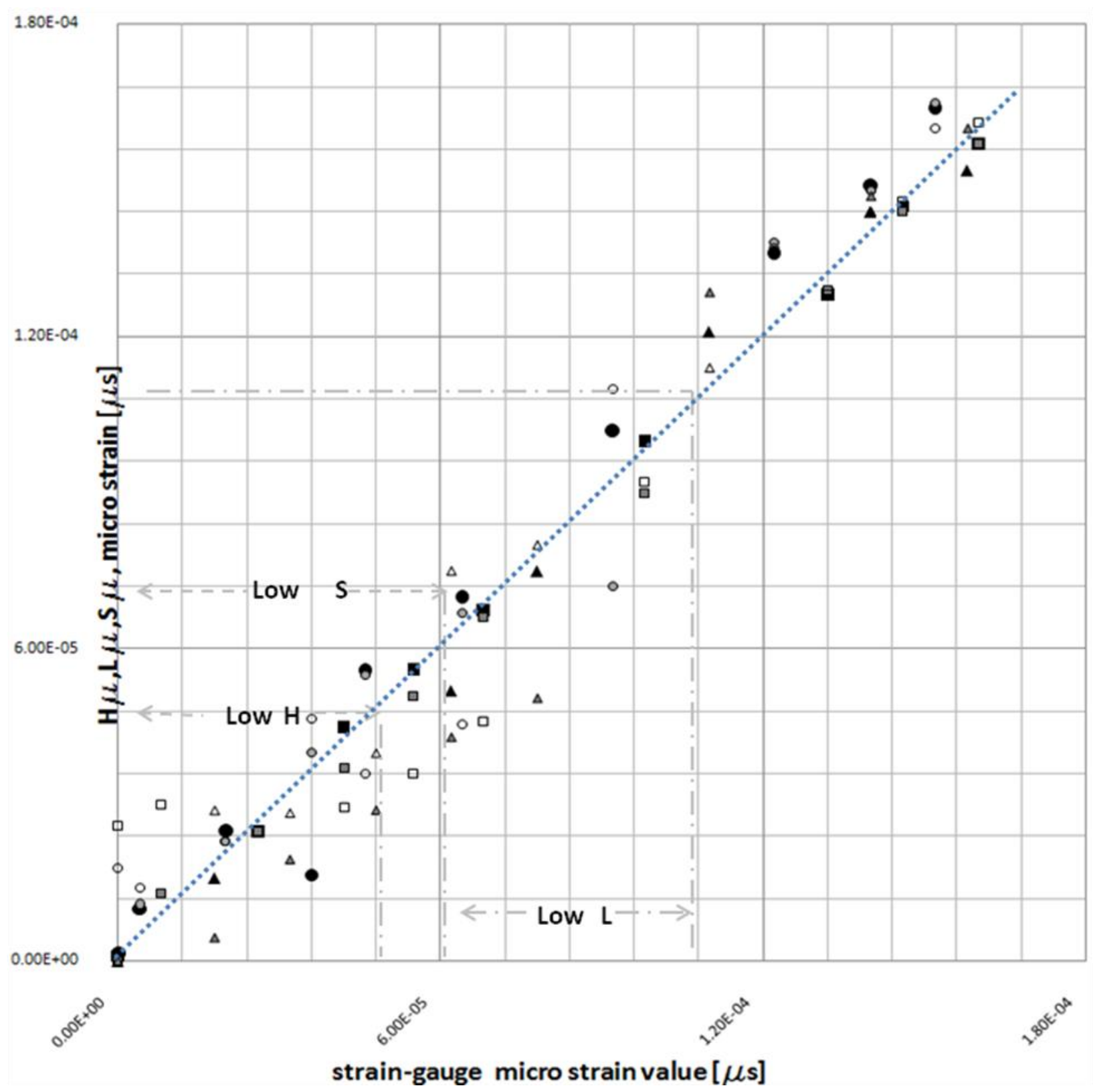

Figure 5.- Strain from chromatic parameters H, L, S as a function of directly measured strain for three separate tests. $\boldsymbol{\bullet}, \boldsymbol{\varnothing}, \bigcirc$ Test 1: Hm, Lm, Sm; $\square, \square, \square$ Test 2: Hm, Lm, Sm; $\triangle$ Test 3: Hm, Lm, Sm

Conclusions. Results have been presented for the variation of three chromatic parameters (H, L, S) with micro-strain, the chromatic parameters being derived from CCD camera images of the interference pattern produced by a photo-elastic element. It has been shown that the variation of these parameters with strain have quasi-cyclical features, each being phase shifted with respect to the others. This has the potential for providing an extended measurement range at optimum sensitivity by automatically selecting the appropriate chromatic parameter most suitable for particular ranges of strain.

Inspection of the raw outputs ( $\mathrm{r}, \mathrm{g}, \mathrm{b})$ from the $\mathrm{R}, \mathrm{G}, \mathrm{B}$ camera pixels indicates that although these outputs also show a wavelength dependent cyclical behavior, the phase differences are considerably less than those observed with the chromatic parameters H, L, S, so making the latter the preferred choice. Other chromatic parameters (e.g. $x=r /(r+g+b), y=g /(r+g+b)$ [7]) also show some cyclical 
behavior but cannot be so conveniently processed as $\mathrm{H}, \mathrm{L}, \mathrm{S}$ to provide range extension and optimum sensitivity. Ongoing work shows that the calculation procedure involving $\mathrm{H}, \mathrm{L}, \mathrm{S}$ described here can be significantly simplified using secondary chromatic processing [9] to produce a linear relationship between chromatic properties and micro-strain.

\section{References}

[1] Perry, C. C., 1984, The resistance strain gauge revisited, Experimental Mechanics, 24(4), pp. $286-299$.

[2] Frew D.J., Forrestal M.J., Chen, W., 2002, Pulse Shaping Techniques for Testing Brittle Materials with a Split Hopkinson Pressure Bar, Experimental Mechanics, 42, pp. 93-106.

[3] Huang H., Asay J.R., 2005, Compressive strength measurements in aluminium for shock compression over the stress range of 4-22 GPa, J. Appl. Phys. 98 (3).

[4] Wallhead I. R., Edwards L., 1997, A practical guide to the measurement of the elastic stress intensity factor in engineering materials by the method of caustics, The Journal of Strain Analysis for Engineering Design, 32 (4), pp. 253-266.

[5] Zandman F., Render S., Dally J., 1977, Photoelastic Coatings, Society for Experimental Mechanics press.

[6] Ramesh K., 2000, Digital Photoelasticity, Springer.

[7] Murphy M. M., Jones G. R., 1992, Polychromatic birefringence sensing for optical fibre monitoring of surface strain, Eurosensors V Conference, 2, pp. 691-695.

[8] Madhu, K.R., Ramesh, K., 2007, Colour Adaptation in Three Fringe Photoelasticity, Experimental Mechanics 47(2) pp. 271-276.

[9] Jones G.R., Deakin A.G., Spencer J.W., 2008, Chromatic Monitoring of Complex Conditions, CRC press.

[10] Information found at http://vishay.com

[11] Deakin A.G. , CIMS, Centre for Intelligent Monitoring Systems, The University of Liverpool.

[12] Allen M. P., 1997, Understanding Regression Analysis, Springer US.

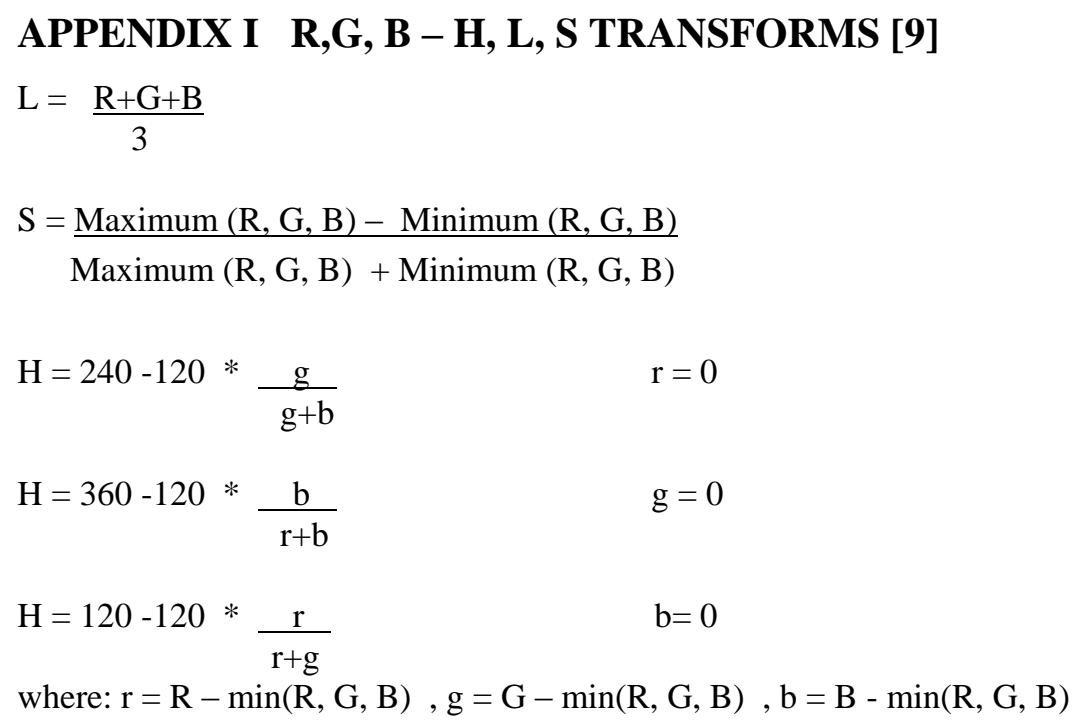

